\title{
Comparison of Multiuser Detectors for Synchronous Flat Rayleigh Fading CDMA Channels
}

\author{
$\mathrm{H}-\mathrm{Y}$. Wu \\ A. Duel-Hallen
}

Center for Communications and Signal Processing Department of Electrical and Computer Engineering North Carolina State University

$$
\begin{aligned}
& \text { TR-94/7 } \\
& \text { April } 1994
\end{aligned}
$$




\title{
Comparison of Multiuser Detectors for Synchronous Flat Rayleigh Fading CDMA Channels
}

\author{
$\mathrm{Hsin}-\mathrm{Yu} \mathrm{Wu}$ and Alexandra Duel-Hallen \\ Department of Electrical and Computer Engineering, Box 7911, \\ North Carolina State University, Raleigh, NC 27695
}

\begin{abstract}
We study performance of several multiuser detection schemes for synchronous CDMA frequency non-selective Rayleigh fading channels. In the first part of the paper, asymptotic multiuser efficiency (AME) of the ideal decision-feedback detector is shown to be unity for any number of users. In the second part, the effect of channel estimation errors on the performance of multiuser detectors is investigated. The average BERs of the two-stage detector and the decorrelator in the presence of channel mismatch are evaluated through analysis and simulations.
\end{abstract}

\section{Summary}

Multiuser detection was shown to be superior to the conventional detection for additive Gaussian noise (AWGN) CDMA channels (see references of [1-4]). In this paper, we investigate the ability of the multiuser detectors to combat flat fading and their performance degradation due to imperfect channel estimation.

In [2], a closed form expression for the average bit error rate (BER) of a two-user ideal decision-feedback detector for synchronous flat Rayleigh fading CDMA channel was derived. Using this result, we also showed that the AME of a two-user decision-feedback detector is unity. In this paper, we consider the AME of an ideal Kuser decision-feedback detector. The average BER of user 1 can be expressed as

$$
P E_{d f}=\sum_{k=1}^{K} \operatorname{Pr}\left(E_{k}\right) \int_{0}^{\infty} P e_{k}(x) f_{\gamma_{1}}\left(x \mid E_{k}\right) d x \text {. }
$$

where $E_{k}$ is the event that user 1 is the $\mathrm{k}$-th strongest user $(1 \leq k \leq K), P e_{k}(x)$ is the error rate of user 1 with $\mathrm{SNR}=\mathrm{x}$, and $f_{\gamma_{1}}\left(x \mid E_{k}\right)$ is the conditional density (pdf) of the SNR. By observing the structure of the conditional pdfs $f_{\gamma_{1}}\left(x \mid E_{k}\right)$, we deduce that the AME of an ideal $\mathrm{K}$-user decision-feedback detector is equal to unity. The conditional pdfs $(\mathrm{k}=1 . . \mathrm{K}-1)$ and $\mathrm{k}=\mathrm{K}$ are in the form of $\prod_{1} \exp \left(-x / \bar{\gamma}_{i}\right) \prod_{j}\left[1-\exp \left(-x / \bar{\gamma}_{j}\right)\right]$ a n d $\prod_{1} \exp \left(-x / \bar{\gamma}_{i}\right)$ respectively. As the average SNR of user $1\left(\bar{\gamma}_{1}\right)$ becomes large, the integrals of the products of the conditional pdfs with the error probabilities $P e_{k}(x)$ (Qfunctions) become insignificant for all $\mathrm{k}$ except for $\mathrm{k}=\mathrm{K}$ (the last term). The average $\mathrm{BER}$ converges to the last integral (i.e., when user 1 is the weakest user), which equals to the single user bound. Thus the AME of the ideal decision-feedback detector is unity for any number of users. Simulations also show that the effect of error propagation is not significant for the actual decision-feedback detector.

We are also interested in investigating the sensitivity of multiuser detectors due to the imperfect channel estimation. The decorrelator with channel mismatch was analyzed in [3]. We focus our analysis on a two-stage detector with the decorrelator as the first stage [4]. Assuming that the fading channel is a first order Markov process, and that the optimal, minimum mean square error channel estimator (Kalman filter) is used, we will determine the average BER with the consideration of the channel mismatch by computer simulations and Stein's unified analysis [3].

\section{References}

[1] A.Duel-Hallen, "Decorrelating DecisionFeedback Multiuser Detector for Synchronous Code-Division Multiple Access Channel," IEEE Transactions on Communications, Vol. COM-41, No. 2, Feb. 1993, pp.285-290.

[2] H.-Y.Wu, A.Duel-Hallen, "Performance of Multiuser Decision-Feedback for Flat Rayleigh Fading Synchronous CDMA Channels," proceedings of CISS, March 1994, Princeton, NJ, to appear.

[3] Z.Zvonar,"Multiuser Detection for Rayleigh Fading Channels," PhD Thesis, Northeastern University, 1993.

[4] M.K.Varanasi, B.Aazhang, "Nearoptimum Detection in Synchronous Code-Division Multiple Access Channel," IEEE Transactions on Communications, Vol. COM-39, No. 5, May 1991, pp. 725-736. 